\title{
Verdad y contingencia en el Tractatus de Wittgenstein
}

Balbina Ferrando Bagán

Universidad de Valencia

balbina.fernando@gmail.com

Mucho se ha escrito sobre la cuestión de la verdad en la bibliografía secundaria acerca del Tractatus. Cualquier estudio que clarifique esta obra en su conjunto lleva a cabo una reflexión en torno a ello. Sin embargo, la gran mayoría de estos libros centra su atención únicamente en la conexión de la teoría de la proposición -esto es, la teoría pictórica del significado y la teoría de las funciones de verdad-con la lógica, de modo que nada o muy poco dice acerca de su relación con la noción de contingencia y las repercusiones que esto tiene en el conjunto de la obra wittgensteiniana. Un ejemplo de ello es el ya clásico estudio Introducción al Tractatus de Wittgenstein de H.O. Mounce ${ }^{1}$. En él, Mounce expone las nociones de verdad y falsedad en los capítulos relativos a la teoría de la proposición con el objetivo último de clarificar la filosofía de la lógica tractariana. Nada más lejos de nuestra intención el defender que Mounce esté errado en su planteamiento de

1 Lo mismo ocurre en otros estudios como Wittgenstein de A. Kenny y El concepto de filosofía en Wittgenstein de K. T. Fann. 
la cuestión. Sostenemos, como él lo hace, que la pretensión última del Tractatus es la de mostrar la estructura profunda de la lógica que subyace a todo lenguaje y los compromisos ontológicos que de ella se deducen. Sin embargo, creemos que es necesario ahondar también en la cuestión de las proposiciones empíricas que, al fin y al cabo, son las portadoras de verdad y las que establecen la relación lenguaje-mundo que posibilitará el significado. Esto quedará más claro con la elaboración que realizaremos a continuación, tras la que concluiremos que en el Tractatus encontramos una caracterización de la verdad como no-necesaria que se mantendrá en la filosofía wittgensteiniana tras el derrumbe de muchos de los presupuestos básicos de su filosofía temprana.

Lo primero que destacaremos es la diferenciación wittgensteiniana entre el estatuto de la ciencia y el de la filosofía $-y$, dentro de ésta, entre el de la lógica y el de la metafísica, siendo la primera su base-. Así, en el Tractatus podemos leer:

\subsection{La filosofía no es ninguna de las ciencias naturales.}

(La palabra «filosofía» ha de significar algo que está por arriba o por debajo, pero no junto a las ciencias naturales).

Como mostraremos, verdad y falsedad serán nociones relativas al ámbito de la ciencia, no cabe predicarlas de las «proposiciones» filosóficas.

En su Tractatus, Wittgenstein trata el problema de la verdad mediante su teoría de la proposición. Esto es así porque «la pregunta de cómo es posible una coordinación de las relaciones es idéntica al problema de la verdad. Porque este último es idéntico a la cuestión de cómo es posible la coordinación de estados de cosas (de uno designante y de uno designado)»(Wittgenstein, 2003: 24/9/1914- 25/9/1914). En 
esta teoría una proposición es una figura del mundo. Pero, ¿qué es una figura? En palabras de Wittgenstein, «una figura es un modelo de la realidad» (Tr., 2.12). Es un constructo humano que se relaciona con la realidad de modo tal que a los objetos de ésta le corresponden los elementos de aquélla. La figura representa cómo se comportan las cosas, y lo hace mediante una relación figurativa que consiste en coordinar los elementos de la figura y las cosas. Si esto es posible es porque figura y realidad comparten una misma forma (Wittgenstein distingue tres formas de la figura que, de menor a mayor grado de abstracción son: la forma de representación, la forma de figuración y la forma lógica). Es de este modo como se da la relación de isomorfismo lenguaje-realidad. La figura representa su sentido (el estado de cosas que describe) y su verdad o falsedad consiste en la correspondencia, el acuerdo o desacuerdo, entre su sentido y la realidad. Veamos ese isomorfismo en mayor profundidad.

En el Tractatus queda patente una concepción de la verdad como correspondencia, cuya base es el isomorfismo del que hemos hablado. Lo primero que cabe destacar para comprenderlo es la distinción que Wittgenstein establece entre nombre, proposición elemental, proposición y lenguaje. Los nombres son los símbolos simples, a ellos corresponderían objetos de la realidad. Ya hemos expuesto cómo la verdad no puede ser relativa a los nombres, sino a las proposiciones. Así, «el nombre significa el objeto» (Tr., 3.203), y una proposición estará compuesta de nombres. Las proposiciones elementales son aquéllas que están directamente conectadas con el mundo, cuya verdad y falsedad no depende de otras proposiciones sino del mundo mismo. Una proposición elemental es «una concatenación de nombres» (Tr., 4.22) que, como ya hemos visto, están en correspondencia con objetos. La correspondencia 
aquí se da entre proposición elemental (que es aquélla que no admite un análisis posterior) y hecho atómico (aquél que no podemos continuar analizando). Una proposición elemental será verdadera si el estado de cosas que designa se da efectivamente y será falsa en caso contrario. Por otro lado, la verdad o falsedad de una proposición depende de los valores de verdad de las proposiciones elementales que la conforman (aparecen aquí las «tablas de verdad» wittgensteinianas). Dicho de otro modo, las proposiciones elementales funcionan como condiciones veritativas de la proposición en la que se hallan. Habría, en este sentido, dos casos extremos: la tautología (necesariamente 〈verdadera〉) y la contradicción (necesariamente (falsa〉). Pero lo cierto es que no podemos decir que sean propiamente verdaderas o falsas, más bien deberíamos decir que carecen de sentido, y esto es así porque no representan ningún estado de cosas posible, no son figuras de la realidad; es decir, la tautología spermite cualquier posible estado de cosas〉 y la contradicción 〈ninguno〉 (Tr. 4.462). Dicho de un modo más prosaico: no digo nada sobre la realidad al afirmar que <llueve o no llueve $>$ porque esto es necesariamente así, siempre. De lo anterior podemos extraer que toda proposición que no se pueda someter al análisis de sus valores de verdad carece de significado, no es propiamente una proposición, porque el sentido aparece solamente en la proposición que representa un estado de cosas -si no es así ésta carece de sentido, esto es, no es verdadera ni falsa-.

Esto nos muestra que Wittgenstein utiliza 〈verdad〉 y 〈falsedad únicamente en el ámbito de la contingencia. La necesidad queda en el ámbito de lo a priori, es decir, en la lógica. La proposición figura al mundo y el modo en como esto sucede no puede ser dicho, solamente puede ser mostrado: «Un enunciado no puede concernir a la estructura lógica del mundo, 
porque para que un enunciado sea en absoluto posible, para que una proposición PUEDA tener SENTIDO, el mundo tiene que tener ya la estructura lógica que tiene. La lógica del mundo es anterior a toda verdad y falsedad» (Wittgenstein, 2003: 18/10/1914). La lógica es lo que posibilita todo lo que puede ser siquiera pensable y es la sustancia para todo mundo posible. De modo que:

5.123 Si un dios crea un mundo en el que determinadas proposiciones son verdaderas, con ello crea también ya un mundo en el que todas las proposiciones que se siguen de ellas son correctas. Y, de modo similar, no podría crear un mundo en el que la proposición $<$ p $>$ fuera verdadera sin crear todos sus objetos.

Así pues, la lógica es lo esencial, ni siquiera un dios podría subvertir las leyes lógicas ni el esquema de contingencia-necesidad que hemos expuesto. Además, tampoco puede alterar la verdad-correspondencia (si un Dios hace que una proposición sea verdadera deberá crear con ella todos sus objetos). Un dios podría tornar verdaderas las proposiciones falsas y falsas las proposiciones verdaderas, porque ése es el ámbito de lo contingente, de lo que podría ser de otro modo. Pero esto muestra que ni siquiera un dios puede hacer necesaria la verdad (pues cabría la posibilidad de que ésta no fuera tal, él mismo podría tornarla falsa) ni contingente la forma lógica, porque es la esencia del mundo ser así -y, dicho sea también, es la esencia porque soy yo la que describe y sólo puedo describir bajo esta forma, no puedo siquiera pensar algo ilógico. Yo estoy a la base, yo abro mundo y lo hago bajo una forma lógica-.

Así, tras todo lo expuesto, estaremos de acuerdo en la siguiente conclusión respecto al problema de la contingencianecesidad y su relación con la verdad: lo que queda fuera de 
juego es la noción de verdad necesaria; y si la verdad no es necesaria, salvo en el caso de la verdad lógica, deberemos replantearnos de qué habla Wittgenstein cuando habla de verdad.

Veamos la definición de verdad que expone Wittgenstein en su Tractatus:

4.063 Una imagen para explicar el concepto de verdad: mancha negra sobre papel blanco; es posible describir la forma de la mancha diciendo de cada punto de la superficie si es blanco o negro. Al hecho de que un punto sea negro le corresponde un hecho positivo; al de que un punto sea blanco (no negro), un hecho negativo [...]. Pero para poder decir que un punto es negro o blanco, tengo que saber antes cuándo a un punto se le llama negro y cuándo se le llama blanco; para poder decir $<\mathrm{p}>$ es verdadero (o falso) tengo que haber determinado en qué circunstancias llamo verdadero a $<$ p $>$, y con ello determino el sentido de la proposición.

Hasta mediados del párrafo parece que estamos leyendo la clásica teoría de la verdad como correspondencia, pero si continuamos podremos observar de qué modo entra en ella el punto de vista del yo. Existiría una verdad-correspondencia pero no absoluta, sería una verdad sesgada por el modo en que describo la realidad y en el que han sido establecidas previamente (antes de mi contacto con lo que es el caso) las condiciones de la descripción, esto lo veremos quizás más claramente con el símil de la red que expondremos a continuación.

Como ya hemos señalado, verdad y falsedad son relativas al ámbito de lo contingente -de lo que podría ser de otro modo- y de la explicación de este ámbito da cuenta la ciencia. Hemos expuesto la teoría del isomorfismo lenguaje-realidad que encontramos en el Tractatus y, tras esto, comprendemos mejor su teoría de la verdad como correspondencia, cons- 
truida sobre este isomorfismo. Veamos cómo ésta se da en el ámbito de las ciencias teniendo en cuenta que tras lo presentado ya podemos intuir que Wittgenstein no defenderá que las verdades científicas sean absolutas. Leemos:

6.341 La mecánica newtoniana, por ejemplo, lleva la descripción del mundo a una forma unitaria. Imaginémonos una superficie blanca con manchas negras irregulares. Diríamos entonces: cualquiera que sea la figura que toma cuerpo así, puedo aproximarme arbitrariamente a su descripción, cubriendo la superficie con una red cuadriculada suficientemente fina y diciendo, acto seguido, de cada cuadrado que es blanco o que es negro. Habré llevado de este modo la descripción de la superficie a una forma unitaria. Esta forma es arbitraria, puesto que con igual éxito hubiera podido utilizar una red con aberturas triangulares o hexagonales [...]. A las diferentes redes corresponden diferentes sistemas de descripción del mundo.

En lo primero que cabe reparar es en que la superficie que vamos a describir es ya considerada como una figura; es decir, en ningún momento Wittgenstein habla de que la realidad en sí sea de tal o cual forma y que tras esto la describamos con una u otra teoría, sino que la realidad que voy a describir mediante la mecánica está ya configurada siempre, la percibo pensándola ya bajo la forma de una figura y a la vez, como veíamos en el parágrafo anterior, la conformo con esta figura. En la ciencia (en las teorías) la relación se establece desde el lenguaje hacia el mundo, no desde el mundo hacia el lenguaje. En este sentido, las reflexiones que elabora Rhees en algunos de sus ensayos acerca del Tractatus resultan muy clarificadoras. Por ejemplo, en The Philosophy of Wittgenstein podemos leer: «I cannot «correlate〉 unless I am 
working with a picture» (Rhees, 1979:40), es decir, no se da el caso de que primero perciba el mundo y después establezca la relación figurativa, esa relación se da mientras percibo el mundo. Además, en esa misma página Rhees afirma: (refiriéndose a los epígrafes 2.1 y 2.11 del Tractatus) «this shows what is meant by picturing facts to ourselves: constructing a situation in a logical space; or we might say, seeing it in a system». Esto, como bien ilustra Rhees, implica que si bien dentro de ese sistema las proposiciones son lógicamente independientes -como han mostrado las diferentes lecturas del Tractatus- esto no significa que no posean relaciones significativas; o lo que es lo mismo, que no encontramos una ruptura total entre el primer y el segundo Wittgenstein, pues ya aparece en el Tractatus la idea de que la verdad (la verdad no lógica, se entiende) lo es siempre dentro de un sistema y que este sistema puede variar.

¿Significa esto que todas las teorías son para Wittgenstein igualmente válidas? La respuesta sería probablemente que no porque, tal y como hemos expuesto, cuando nos relacionamos con el mundo lo hacemos ya desde un sistema. No establecemos la relación figurativa en el vacío, no existe una realidad independiente de la forma de figuración. La pregunta en sí es un sinsentido porque pretende quedar planteada fuera de la forma de figuración, lo cual sería un imposible $^{2}$. ¿Qué es entonces lo que nos permite escoger entre diversas teorías? Wittgenstein propondrá la sencillez como criterio (Tr., 6.341, 6.342, 6.363).

2 Cabe destacar que el autor no responde directamente a esa pregunta en el Tractatus. 
Solamente existe una necesidad lógica (Tr. 6.37), porque todo lo que proviene de nuestra experiencia podría ser de otro modo (Tr. 5.634). De manera que:

6.371 A toda la visión moderna del mundo subyace el espejismo de que las llamadas leyes de la naturaleza son las explicaciones de los fenómenos de la naturaleza.

6.372 Y así se aferran a las leyes de la naturaleza como a algo intocable, al igual que los antiguos a Dios y al destino.

Y ambos tienen razón y no la tienen. Pero los antiguos son, en cualquier caso, más claros en la medida en que reconocen un final claro, en tanto que en el nuevo sistema ha de parecer como si todo estuviera explicado.

Queda claro entonces, el estatuto de la ciencia para Wittgenstein $y$, puesto que la verdad es siempre relativa al ámbito de la ciencia, queda claro con esto el estatuto de la verdad. Lo importante, lo esencial, no es que la proposición sea verdadera o falsa, sino que la proposición sea. Su posibilidad de ser es su sentido. Y precisamente esto es lo que podemos conocer a priori: su posibilidad que será, además, lógica.

Podemos observar, pues, un doble juego del término verdad. Teniendo en cuenta que lenguaje y mundo se dan a la vez -como bien advierte Rhees criticando el Companion de Black «it is pointless to say, as Black does: we must have some view of what reality is like, before we can ask if the symbolism is adequate to describe it» (Rhees, 1970:23)- y que se establece entre ellos una relación de correspondencia, advertimos al respecto dos niveles. El primero es el nivel esencial y lógico; el segundo es el empírico, que es contingente. De modo que la lógica posee verdades intocables y definitivas porque «la lógica no es una teoría sino una figura 
especular del mundo» (Tr. 6.13). Como veíamos, mundo y lenguaje poseen la misma forma lógica, a nivel lógico son iguales (no hay uno más esencial que el otro, lo esencial es la lógica que ambos comparten), es en este sentido que los límites de uno son los límites del otro. Habría, pues, una correspondencia lógica sin la cual es imposible predicar nada del mundo (y esta teoría de la correspondencia pondría en juego la verdad propia del punto de vista del objetivismo). Pero, por otro lado, encontramos esa verdad contingente -la verdad empírica sobre la que hemos reflexionado- que aparece siempre dentro de la teoría de la que forma parte y que persistirá en las obras del maduro Wittgenstein.

En conclusión, encontramos en el Tractatus un doble uso de la noción de verdad que, a nuestro entender, no ha sido correctamente interpretada en la mayoría de los estudios sobre Wittgenstein. Si bien es cierto que encontramos una verdad necesaria -la relativa a la lógica-, también encontramos una verdad contingente -la científica, relativa al marco desde el que es formulada-. Frente a las lecturas que han visto en el Tractatus una defensa del realismo metafísico (en el que el lenguaje reflejaría los hechos brutos del mundo, el mundo ya hecho, el mundo tal y como es con independencia del yo que conoce), observamos un cierto relativismo conceptual en el que la verdad sólo sería tal dentro del sistema teórico desde el que es concebida, un sistema que puede variar. Esto muestra la vinculación al respecto entre el primer y el segundo Wittgenstein: el Tractatus ya adopta algunos de los principios del relativismo conceptual que aparecerán en sus escritos posteriores. 
Bibliografía

Black, M. (1964). A companion to Wittgenstein's 〈Tractatus〉, Cambridge: Cambridge University Press.

Fann, K.T. (1975). El concepto de filosofía en Wittgenstein. M.A. Bertrán (tr.). Madrid: Tecnos.

Kenny, A. (1984). Wittgenstein. A. Deaño (tr.). Madrid: Alianza Editorial.

Mounce, H.O. (1993). Introducción al 〈Tractatus〉 de Wittgenstein. J. Mayoral y P. Vicente (tr.). Madrid: Tecnos.

Rhees, R. (1970). 〈Ontology〉 and Identity in the Tractatus à propos of Black's Companion. En R. Rhees. Discussions of Wittgenstein. London: Routledge \& Kegan Paul.

Wittgenstein, L. (2003). Cuadernos de notas (1914-1916). J.D. Mateu (tr.). Madrid: Síntesis.

Wittgenstein, L. (2003). Tractatus logico-philosophicus. J. Muñoz e I. Reguera (tr.). Madrid: Alianza Editorial. 
Resumen

Tradicionalmente se ha leído el Tractatus como una obra que sostiene una posición de realismo metafísico. Frente a esto, defenderemos que en esta obra ya aparecen algunos puntos cercanos al relativismo conceptual que entroncan con su filosofía segunda. Para ello, expondremos la concepción de la verdad (centro de la discusión en torno al relativismo) que encontramos en el Tractatus, observando el doble uso de esta noción que allí aparece: la verdad lógica -esencial y necesaria- y la verdad epistemológica -relativa al marco desde el que es formulada-.

Palabras clave: Wittgenstein, relativismo, verdad, lógica, epistemología.

\section{Abstract}

The Tractatus has been read as a work that holds a metaphysical realism position. Against this, we will defend that in this same work there is already some close point to the conceptual relativism that tie in with his second philosophy. In order to achieve this, the conception of truth (center of the discussion regarding relativism) that we found on the Tractatus will be exposed, analyzing the double use of this notion that lays there: logical truth -essential and necesary- and epistemological truth -related to the frame that is formulated-.

Key words: Wittgenstein, relativism, truth, logic, epistemology. 